\title{
Bacterial colonies detecting and counting based on enhanced CNN detection method
}

\author{
Shousheng Liu ${ }^{1,2,3}$, Zhigang Gai ${ }^{1,2,3, *}$, Xu Chai ${ }^{1,2,3}$, Fengxiang Guo ${ }^{1,2,3}$, Mei Zhang ${ }^{1,2,3}$, Shanshan Xu ${ }^{1,2,3, *}$, Yibao Wang ${ }^{1,2,3}$, \\ Ding $\mathrm{Hu}^{1,2,3}$, Shaoyan Wang ${ }^{1,2,3}$, Lili Zhang ${ }^{1,2,3}$, Xueyu Zhang ${ }^{1,2,3}$, Zhigang Chen ${ }^{1,2,3}$, Xiaoling Sun ${ }^{1,2,3}$, and Xin Jiang ${ }^{1,2,3}$ \\ ${ }^{1}$ Qilu University of Technology (Shandong Academy of Sciences), Institute of Oceanographic Instrumentation, 37 Miaoling Road, \\ Qingdao, China, 266100 \\ ${ }^{2}$ Shandong Provincial Key Laboratory of Marine monitoring instrument equipment technology, 37 Miaoling Road, Qingdao, China, \\ 266100 \\ ${ }^{3}$ National Engineering and Technological Research Center of Marine Monitoring Equipment, 37 Miaoling Road, Qingdao, China, \\ 266100
}

\begin{abstract}
Bacterial colonies detecting and counting is tedious and time-consuming work. Fortunately CNN (convolutional neural network) detection methods are effective for target detection. The bacterial colonies are a kind of small targets, which have been a difficult problem in the field of target detection technology. This paper proposes a small target enhancement detection method based on double CNNs, which can not only improve the detection accuracy, but also maintain the detection speed similar to the general detection model. The detection method uses double CNNs. The first CNN uses SSD_MOBILENET_V1 network with both target positioning and target recognition functions. The candidate targets are screened out with a low confidence threshold, which can ensure no missing detection of small targets. The second CNN obtains candidate target regions according to the first round of detection, intercepts image sub-blocks one by one, uses the MOBILENET_V1 network to filter out targets with a higher confidence threshold, which can ensure good detection of small targets. Through the two-round enhancement detection method has been transplanted to the embedded platform NVIDIA Jetson AGX Xavier, the detection accuracy of small targets is significantly improved, and the target error detection rate and missed detection rate are reduced to less than $1 \%$.
\end{abstract}

\section{Introduction}

Bacterial colonies detecting and counting is tedious and time-consuming work. Someone researchers put forward some semi-automatic method like Histograms of Oriented Gradient (HOG)[1], this kind of method often require more multifarious image pre-processing step, so the degree of automation is low with lack of efficiency.

In recent years, deep learning target detection methods based on $\mathrm{CNN}$ for automatic feature extraction have gradually replaced traditional target detection methods. The deep learning approach has gone through two stages of development. The first stage is the twostep method of candidate region plus deep learning classification. The candidate region is extracted and the corresponding region is classified with the deep learning method, such as R-CNN, Fast R-CNN, Faster R-CNN[2]. The second stage is a one-step method based on deep learning regression. Such an algorithm does not need to extract candidate regions separately, but also puts the recognition of regions into deep learning, and realizes end-to-end target detection, such as YOLO/SSD[3] and other methods.

Small target detection is always a difficult problem in deep learning $\mathrm{CNN}$ model. The target detection algorithm has undergone a series of improvements from traditional to deep learning, and has made considerable progress in the accuracy and real-time performance of target detection. However, there still exists the problem of poor accuracy of small target recognition. Most of the small target detection method is based on the general regular medium target oriented method, such as classic SSD single step method, two step Faster-RCNN, these methods mainly for general purpose data set to design solutions that are good at normal target detection, but not sufficient for small target detection[4].

\section{Target Detection Method Based On Cnns}

Because the bacterial colonies have the characteristics of small proportion of target in image, SSD_MOBILENET_V1 is adopted as the front-end target detection network. Because high confidence threshold will miss small target, so we try lower threshold, which may result in too much error detection which is not colony. Behind the output end of the detection network, the MOBILENET_V1[5] is applied to remove error detection at the cost of relatively low

\footnotetext{
* Corresponding author: zhiganggai@126.com (Z.Gai); xss_qd@126.com (S. Xu)
} 
computation. Procedure of colonies detection by $2 \mathrm{CNNs}$ is shown in Fig1.

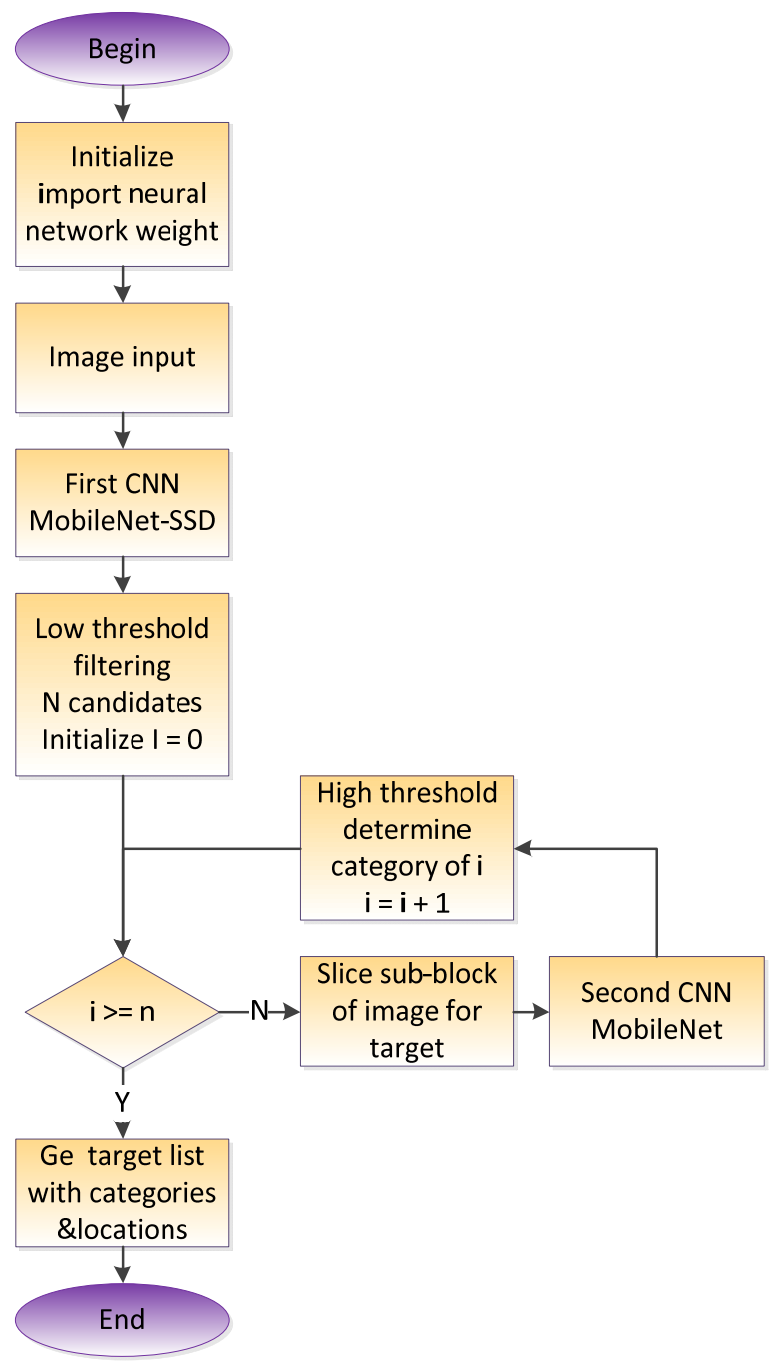

Fig. 1. procedure of colonies detection by $2 \mathrm{CNNs}$

\subsection{MOBILENET_V1 CNN recognition}

MOBILENET_V1 is a CNN suitable for mobile terminals proposed by Google in 2017. The main innovation is that on the basis of standard VGG, deep separable convolution depthwise separable convolution is used to decompose convolution kernels to reduce computation. The model introduces two superparameters, the first is width multiplier $\alpha$, which is used to reduce the number of feature maps to reduce the thickness of the model; the second is resolution multiplier $\rho$, which is used to reduce the resolution of the image, that is, to reduce the size of feature map and further reduce the amount of calculation.

\subsection{SSD_MOBILENET_V1 CNN detection}

The detection method SSD_MOBILENET V1 is developed from SSD, Which is improved on the basis of YOLO. SSD has no window generation process. Each neuron on the Feature Map of different scales predicts $\mathrm{N}$ candidate windows. The sensory field of neurons on larger Feature Map is smaller, so the corresponding Anchors of neurons on larger Feature Map are smaller, and vice versa. The common feature of SSD and YOLO is that each neuron on Feature Map is used to predict $n$ windows, which is a good transition between regressionbased method and candidate-based method. SSD_MOBILENET_V1 replaces the VGG feature extraction network of SSD by MOBILENET_V1.

\section{Dataset preparation and train}

\subsection{Collecting pictures of RGB images}

We took pictures of RGB image for bacterial colonies in multiple culture medium. In order to meet the training requirements, the total number of targets contained in the training data provided must meet certain requirements.

\subsection{Label and train MOBILENET_V1}

Besides of pictures that have lots of colonies vary from 5-100, we also took pictures of RGB image for empty culture medium. We set label 1 for all images with colonies, then set label 0 for all images without any colony. Those images and labels are prepared for training of recognition network MOBILENET_V1.

\subsection{Label and train SSD_MOBILENET_V1}

In view of the acquisition of each dish colony images, one by one manually mark location of the colony marked results deposit with petri dish image XML annotation files of the same name, each annotation file corresponds to a petri dish image, annotation files inside is the location of the colony properties, mark each object file label represents a colony rectangular area in the upper left and lower right pixel coordinates a pixel coordinate. Based on the pre-training model, the colony dataset is used for the tuning training. When the number of iterations reaches 200,000, the training loss tends to be stable with a value of 0.9 . After the training process is completed, the network parameters that have been trained are saved and used to detect colonies in test image.

\section{Experiments and results analysis}

\subsection{Detect by SSD_MOBILENET_V1}

The first CNN uses the SSD_MOBILENET_V1 detection network. The super-parameters width multiplier is reduced from 1.0 of the standard to 0.5 . The detection time is reduced to $1 / 4$. The images to be tested are sent to the first $\mathrm{CNN}$ to perform the inference process, and the first $\mathrm{CNN}$ outputs the detection results. In the result queue, the detection targets are ranked from high to low according to the confidence probability value, and the candidate targets are filtered out with a low confidence threshold T1(0.1). 
The candidate target regions are obtained according to the first $\mathrm{CNN}$, and the target image sub-blocks are intercepted one by one, so as to improve the target pixel proportion. The small target with a relatively small pixel proportion $(<1 \%)$ is transformed into a large target with a relatively high pixel proportion $(>50 \%)$.

\subsection{Recognize by MOBILENET_V1}

The second CNN uses the MOBILENET_V1 recognition network with only the target recognition function, and the computing time of the recognition network is $1 / 8$ of the baseline detection network. Suppose the second CNN recognizes 4 targets and the computing time of the recognition is $1 / 2$.

We put target sub-block pixel image one by one into the second CNN to perform inference process, then we get confidence score from high to low by the destination queue. With another threshold value (0.85-0.95), if target confidence is higher than the threshold, we confirm that it is real target, otherwise it is error target.

\subsection{Run on NVIDIA Jetson AGX Xavier}

NVIDIA Jetson AGX Xavier is the latest generation of NVIDIA industry leading embedded Linux high performance computer, mainly including an 8-core NVIDIA Carmel ARMv8.2 64-bit CPU, made up of 8 stream multi-processors 512 core Volta architecture GPU, support parallel computing language CUDA[6] 10, support multi-precision computing, FP16 computing power is 11 TFLOPS (floating-point operations per second), INT8 is 22 TOPS.

The Xavier platform comes with the full NVIDIA JetPack SDK for AI development, including the latest versions of CUDA, cuDNN, and TensorRT. These development software is very convenient to use, plus Xavier platform powerful reasoning computing power, Xavier will shine in the manufacturing, logistics, retail, service and other edge computing AI application scene.

Firstly, we build GPU version of caffe[7] deep learning framework. Then we run target detection network SSD_MOBILENET V1 and recognition network MOBILENET_V1 on NVIDIA Jetson AGX Xavier and get running speed above 20 fps. The detecting and counting result of colonies is shown in Fig. 2 below.
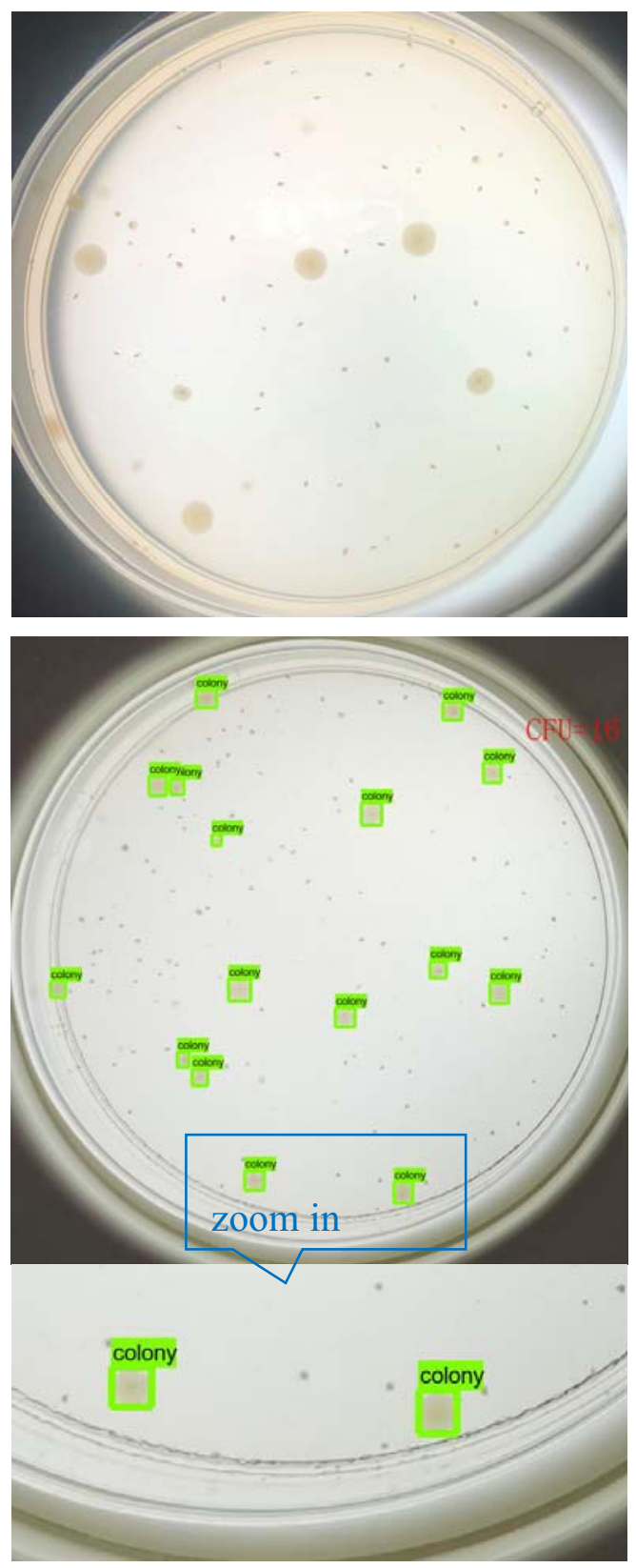

Fig. 2. input and output of $2 \mathrm{CNNs}$ detection for colonies.

\section{Conclusion}

In order to find a solution to the bacterial colonies detecting and counting problem, we propose a small target enhancement detection method based on double CNNs, which can not only improve the detection accuracy, but also maintain the detection speed similar to the general detection model. The detection method uses double CNNs. The first CNN uses SSD_MOBILENET_V1 network with both target positioning and target recognition functions. The candidate targets are screened out with a low confidence threshold, which can ensure no missing detection of small targets. The second $\mathrm{CNN}$ obtains candidate target regions according to the first, intercepts image subblocks one by one, uses the MOBILENET_V1 network containing only target recognition function, and screens out targets with a higher confidence threshold, which can 
ensure good detection of small targets. Through the tworound enhancement detection method proposed by the invention and transplanted to the embedded platform NVIDIA Jetson AGX Xavier, the detection accuracy of small targets is significantly improved, and the target error detection rate and missed detection rate are reduced to less than $1 \%$.

We acknowledge support from the National Science Foundation for Youths of China (Grant No. 41806112 and 51802179), Key Research and Development Program of Shandong Province(International Science and Technological Cooperation) (Grant No. 2019GHZ005), Major Scientific and Technological Innovation Program of Shandong Province (Grant No. 2019JZZY020302), Excellent Youth Innovation Team of Shandong Province (Grant No. 2019KJA005), Shandong Province Science Foundation for Youths (Grant No. ZR2018LE005), Shandong Youth Foundation of Academy of Sciences (Grant No. 2019QN0037).

\section{References}

1. DALAL N, TRIGGS B. "Histograms of Oriented Gradients for Human Detection", Proc. of the IEEE Computer Society Conference on Computer Vision and Pattern Recognition. Washington, USA: IEEE, 2005: 886-893

2. Ren S, He K, Girshick R, et al. "Faster R-CNN: Towards Real-Time Object Detection with Region Proposal Networks". IEEE Transactions on Pattern Analysis \& Machine Intelligence, 2017, 39(6):11371149

3. LIU W, ANGUELOV D, ERHAN D, et al. "SSD: Single Shot Multibox Detector". Proc. of the 14th European Conference on Computer Vision. New York, USA: Springer, 2016, I: 21-37

4. Shousheng Liu, Zhigang Gai, Lin Cao, Hui Li, Ding $\mathrm{Hu}$, Fengxiang Guo, and Huimin Qiu "Research on automatic detection method of infrared marine buoy based on CNN", Proc. SPIE 11427, Second Target Recognition and Artificial Intelligence Summit Forum, 2020

5. Howard A G, Zhu M, Chen B, et al. "MobileNets: Efficient Convolutional Neural Networks for Mobile Vision Applications"[J]. arXiv: Computer Vision and Pattern Recognition, 2017

6. Ortega L, Rueda A. Parallel drainage network computation on CUDA[J]. Computers \& Geoences, 2010, 36(2):171-178

7. Yangqing Jia, Evan Shelhamer, Jeff Donahue, Sergey Karayev and etc. Caffe: Convolutional Architecture for Fast Feature Embedding. In Proceedings of the 22nd ACM international conference on Multimedia (MM '14). NY, USA, 2014: 675-678 